Revista de Matemática: Teoría y Aplicaciones 2018 25(2) : 293-318

CIMPA - UCR ISSN: 1409-2433 (PRINT), 2215-3373 (ONLINE)

DOI: https://doi.org/10.15517/rmta.v25i2.33700

\title{
MODELO PARA LA TRANSMISIÓN DEL VIH EN UNA POBLACIÓN CON DIFERENCIACIÓN DE SEXOS Y USOS DE MEDIDAS PREVENTIVAS
}

\author{
MODEL FOR HIV TRANSMISSION IN A \\ POPULATION WITH SEX DIFFERENTIATION \\ AND USE OF PREVENTIVE MEASURES
}

\author{
HERnÁn Darío TORO-ZAPATA* \\ José Luis CALDERón-GutiérReZ ${ }^{\dagger}$ \\ Óscar Emilio Molina-Díaz
}

Received: 30/Aug/2017; Revised: 18/May/2018; Accepted: 24/May/2018

\begin{abstract}
Revista de Matemática: Teoría y Aplicaciones is licensed under a Creative Commons Reconocimiento-NoComercial-Compartirigual 4.0 International License.

Creado a partir de la obra en http://www.revistas.ucr.ac.cr/index.php/matematica

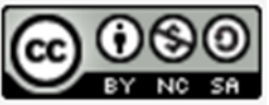

* Licenciatura en Matemáticas, Universidad del Quindío, Quindío, Colombia. E-Mail: hdtoro@uniquindio.edu.co

${ }^{\dagger}$ Facultad de Ciencias de la Administración, Universidad del Valle, Valle del Cauca, Colombia. E-Mail: calderon.jose@univalle.edu.co

${ }^{\ddagger}$ Misma dirección que/Same address as: H.D.Toro. E-Mail: omolina@uniquindio.edu.co
\end{abstract}




\title{
Resumen
}

En este artículo se formula un modelo basado en ecuaciones diferenciales ordinarias, para describir la dinámica de transmisión del VIH considerando una diferenciación en la población según el género y la orientación sexual, con el fin de analizar el impacto que el uso de medidas preventivas tiene en la reducción de la transmisión del virus. Se realiza además un estudio de simulación numérica para ilustrar diferentes escenarios de control mediante medidas preventivas.

Palabras clave: sistema dinámico; estabilidad local; VIH; SIDA; medidas preventivas.

\begin{abstract}
A model based on ordinary differential equations to describe the dynamics of HIV transmission, considering differentiation in the population according to gender and sexual orientation is proposed, in order to analyze the impact of preventive measures in reducing HIV transmission. A numerical study is carried out to illustrate different control scenarios through preventive measures.
\end{abstract}

Keywords: dynamical systems; local stability; HIV; AIDS; preventive measures.

Mathematics Subject Classification: 93C15, 37N25, 92C60.

\section{Introducción}

Desde su aparición en la segunda mitad del siglo XX, la infección por el Virus de Inmunodeficiencia Humana (VIH) y el Síndrome de Inmunodeficiencia Adquirida (SIDA) se han convertido en un problema de salud a escala mundial, y de hecho, constituyen la primera epidemia del siglo XXI. ONUSIDA en su hoja informativa de junio de 2017, reporta a nivel global que 20,9 millones de personas tenían acceso a la terapia antiorretroviral, 36,7 millones [30,8 millones-42,9 millones] de personas vivían con el VIH en 2016 en todo el mundo. 1,8 millones [1,6 millones- 2,1 millones] de personas contrajeron la infección por el VIH en 2016. 1 millón [830.0001,2 millones] de personas fallecieron a causa de enfermedades relacionadas con el SIDA en 2016. 76,1 millones [65,2 millones-88,0 millones] de personas contrajeron la infección por el VIH desde el comienzo de la epidemia y 35,0 millones [28,9 millones-41,5 millones] de personas fallecieron a causa de enfermedades relacionadas con el sida desde el comienzo de la epidemia [33]. 
El reporte de datos de Onusida para 2017 [47], estima para Colombia que el número de nuevas infecciones por VIH se ha venido reduciendo con 7800 nuevos casos en 2005, frente a 6900 en 2010 y 5600 en 2016. La incidencia de VIH por cada 1000 habitantes se estimó entre 0.08 y 0.16. Particularmente de la población de jóvenes de 15-24 años, se estimó un conocimiento de la prevención por parte del $30.2 \%$ de la población.

El uso de los preservativos como método de barrera sigue siendo el mejor método y el más generalizado para evitar el contagio con el VIH. Se hace una distinción entre la eficacia y la efectividad de los preservativos, la eficacia se refiere a la protección recibida bajo las condiciones ideales y las propiedades del preservativo; y la efectividad es definida como la protección recibida bajo las condiciones reales, las propiedades del preservativo y el comportamiento del usuario; por lo tanto, la efectividad de un preservativo es un término usado para referirse al nivel de protección contra la infección del VIH, y otras enfermedades de transmisión sexual (ETS), cuando los preservativos se usan consecuente y correctamente [36].

Según ONUSIDA en sus datos para Colombia, el uso del condón, por lo menos en las relaciones sexuales de mayor riesgo, alcanzó para los hombres un $70.5 \%$, mientras que para el uso de condón en las mujeres no se reporta dato alguno. En la población de hombres gay y otros hombres que tienen sexo con hombres, la población estimada es de 545042 , con una prevalencia de $\mathrm{VIH}$ del $17 \%$ y un uso del condón también del 17\%. La población transgénero se estima en el reporte en 14 608, con una prevalencia de $21.4 \%$ y un uso del condón que alcanza el $91.3 \%$ [47].

Los preservativos de látex para los hombres son esencialmente impermeables a partículas del tamaño de los patógenos que causan ETS, incluyendo la infección VIH, mientras que los estudios sobre la eficacia y efectividad de los preservativos poliuretano para mujeres, han demostrado que estos proveen una barrera efectiva contra organismos inclusive más pequeños que los que causan ETS y es por lo menos equivalente al preservativo para hombres en la prevención de la gonorrea, la tricomoniasis y la clamidia.

El modelado matemático de la dinámica de transmisión del VIH se ha enfocado desde diferentes aspectos de la enfermedad y mediante diversas herramientas matemáticas, como las ecuaciones diferenciales ordinarias y parciales, los modelos discretos, modelos estadísticos y problemas de optimización, entre otros. Se reportan unos pocos estudios que sirven como antecedente y se han enfocado al estudio de modelos matemáticos sobre enfermedades infecciosas [37, 6, 9, 29, 45]. Modelos matemáticos 
para la dinámica de transmisión del VIH [7, 20, 19, 21, 24, 42, 28, 32, 34, $38,41,43,44,50]$. Modelos matemáticos para la prevención, diagnóstico o tratamiento antirretroviral del VIH $[3,48,10,11,26,46]$. Modelos del VIH en poblaciones de riesgo $[1,14,39,45]$. Distribución espacial de la infección $[13,49]$. Problemas de control óptimo en enfermedades infecciosas $[4,5,12,15,23,30,31,40]$. Modelos matemáticos de control óptimo sobre el VIH/SIDA [8, 22, 25].

En este trabajo se formula un modelo matemático que permite determinar la proporción de adopción que deben tener los preservativos en una población con el fin de controlar la transmisión de la enfermedad. En vista de que los comportamientos sexuales de la población son heterogéneos y que el uso del condón es claramente diferenciable en poblaciones homosexuales y heterosexuales, agravado lo anterior a la falta de información precisa, se tiene que también los riesgos de infección son diferenciables según el tipo de acto sexual considerado [27]. En ese sentido, se ha optado por considerar una población bisexual, donde las vías de transmisión se restringen al contacto sexual heterosexual y homosexual entre hombres. En cualquiera de los casos, se asume que las tasas de transmisión y uso del condón son diferentes.

\section{Formulación del modelo}

Se formula un modelo para la dinámica de transmisión del VIH considerando una población bisexual y diferenciada según género, con el fin de estudiar el impacto que el uso de medidas preventivas tiene en la reducción de la transmisión del virus. De manera específica, se modela la transmisión del VIH por vía sexual en una población de mujeres sexualmente activas, susceptibles al contagio $x_{1}=x_{1}(t)$ y hombres sexualmente activos susceptibles al contagio $y_{1}=y_{1}(t)$. Se supone acá que las mujeres sólo adquieren el virus por contacto sexual con hombres infectados (relaciones heterosexuales), debido a que las probabilidades de transmisión en relaciones entre mujeres son en esencia muy bajas [2]. Por el contrario, se supone que los hombres pueden adquirir el virus por relaciones sexuales tanto con mujeres como con hombres, es decir que la población se supone bisexual. Una vez una mujer adquiere el virus, pasa a la categoría de infectada sin diagnosticar $x_{2}=x_{2}(t)$, mientras que los hombres que adquieren el virus pasan al estado de infectado sin diagnosticar $y_{2}=y_{2}(t)$.

Rev.Mate.Teor.Aplic. (ISSN print: 1409-2433; online: 2215-3373) Vol. 25(2): 293-318, Jul-Dec 2018 
Son muchos los factores que pueden dificultar el diagnóstico con el virus, tanto de índole fisiológico como social, lo que hace difícil estimar el tiempo que transcurre desde el momento del contagio hasta el diagnóstico [43], sin embargo, una vez esto ocurre, las mujeres pasan a la categoría de infectadas diagnosticadas $x_{3}=x_{3}(t)$ y los hombres pasan a la categoría de infectados diagnosticados $y_{3}=y_{3}(t)$. Adicionalmente, se supone que todos los individuos diagnosticados se encuentran bajo algún esquema de terapia antiviral, lo que permite asumir que las tasas de transmisión debidas a individuos diagnosticados son muy bajas, y se desprecian en este estudio; es decir, se asume que la transmisión del virus se debe exclusivamente a los infectados sin diagnosticar, como se describe mas abajo.

El modelo está circunscrito a una población con características específicas de homogeneidad y no a una población general, en ese sentido, para modelar la transmisión se consideró que la fuerza de infección obedece al principio de acción de masas; es decir, que la transmisión del virus ocurre sólo cuando se da un contacto efectivo, que corresponde a un encuentro entre un individuo susceptible con uno infectado con una tasa de transmisión que recoge, entre otros aspectos, la probabilidad de que el virus sea transmitido. Se asumen tres tasas de transmisión, a saber: $\beta_{1}$ para el contagio de una mujer susceptible por contacto con un hombre infectado $\left(\beta_{1} x_{1} y_{2}\right) ; \beta_{2}$ para el contagio de un hombre susceptible por contacto con una mujer infectada $\left(\beta_{2} x_{2} y_{1}\right)$ y finalmente, $\beta_{3}$ para el contagio de un hombre susceptible por contacto con un hombre infectado $\left(\beta_{3} y_{1} y_{2}\right)$. Se consideran tres tasas de transmisión apoyados en el hecho de que diferentes prácticas sexuales conllevan a diferentes niveles de riesgo. En la Figura 1 se puede observar una descripción gráfica de los flujos considerados en la construcción del sistema de ecuaciones diferenciales que describe la dinámica y una descripción, las variables y parámetros usados en el modelo se pueden apreciar en la Tabla 1.

El modelo se desarrolla teniendo en cuenta que la población total no es constante; es decir, que esta presenta tasas de mortalidad tanto por causas ajenas al virus, como también relacionadas con el virus y tanto en personas diagnosticadas como sin diagnosticar. En particular, se asume las poblaciones infectadas pueden morir tanto por causas relacionadas con el virus como por causas ajenas a este, esto se apoya en el hecho de que la infección por VIH es crónica, es decir que una persona infectada puede vivir una vida relativamente plena y por mucho tiempo, sin sufrir los efectos de enfermedad. 


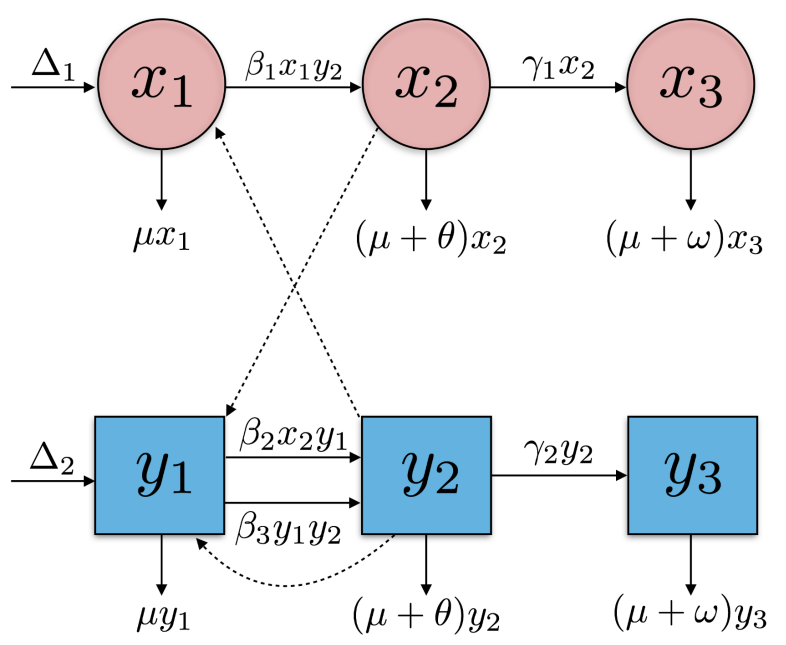

Figura 1: Flujograma que describe gráficamente los flujos considerados en la construcción del sistema de ecuaciones diferenciales para la dinámica.

Se denota con $\Delta_{1}>0$ y $\Delta_{2}>0$ a las tasas de crecimiento constantes de las subpoblaciones de mujeres y hombres, respectivamente, que corresponde a personas que inician su actividad sexual.

El modelo no considera poblaciones de niños ni transmisión vertical del virus. Las consideraciones descritas dan origen al modelo en ecuaciones diferenciales ordinarias:

$$
\left\{\begin{aligned}
\frac{d x_{1}}{d t} & =\Delta_{1}-\beta_{1}\left(1-u_{1}\right) x_{1} y_{2}-\mu x_{1} \\
\frac{d x_{2}}{d t} & =\beta_{1}\left(1-u_{1}\right) x_{1} y_{2}-\delta_{1} x_{2} \\
\frac{d x_{3}}{d t} & =\gamma_{1} x_{2}-\delta_{2} x_{3} \\
\frac{d y_{1}}{d t} & =\Delta_{2}-\beta_{2}\left(1-u_{1}\right) x_{2} y_{1}-\beta_{3}\left(1-u_{2}\right) y_{1} y_{2}-\mu y_{1} \\
\frac{d y_{2}}{d t} & =\beta_{2}\left(1-u_{1}\right) x_{2} y_{1}+\beta_{3}\left(1-u_{2}\right) y_{1} y_{2}-\delta_{3} y_{2} \\
\frac{d y_{3}}{d t} & =\gamma_{2} y_{2}-\delta_{2} y_{3} .
\end{aligned}\right.
$$


Tabla 1: Descripción de las variables de estado y su valor inicial y de los parámetros del modelo y el valor adoptado para su simulación. MSA $=$ Mujeres Sexualmente Activas, HSA $=$ Hombres Sexualmente Activos, $\mathrm{MP}=$ Medidas Preventivas. Los valores de parámetros son estimados de fuentes secundarias, en particular, $\beta_{1}, \beta_{3}$ y $\beta_{3}$ son obtenidos de los rangos propuestos en [27].

\begin{tabular}{l|ll}
\hline \hline Par. & Descripción & Valor \\
\hline$x_{1}$ & MSA susceptibles de adquirir el VIH en un tiempo $t$ & 250 \\
$x_{2}$ & MSA portadoras del VIH no diagnosticadas en un tiempo $t$ & 0 \\
$x_{3}$ & MSA portadoras del VIH diagnosticadas en un tiempo $t$ & 0 \\
$y_{1}$ & HSA susceptibles de adquirir el VIH en un tiempo $t$ & 249 \\
$y_{2}$ & HSA portadores del VIH no diagnosticados en un tiempo $t$ & 1 \\
$y_{3}$ & HSA portadores del VIH diagnosticados en un tiempo $t$ & 0 \\
$\Delta_{1}$ & Mujeres que ingresan a la población sexualmente activa & 1 \\
$\Delta_{2}$ & Hombres que ingresan a la población sexualmente activa & 1 \\
$\beta_{1}$ & Tasa de transmisión hombre a mujer & 0.0006 \\
$\beta_{2}$ & Tasa de transmisión mujer a hombre & 0.0007 \\
$\beta_{3}$ & Tasa de transmisión hombre a hombre & 0.002 \\
$\gamma_{1}$ & Tasa de diagnóstico para las mujeres & 0.1 \\
$\gamma_{2}$ & Tasa de diagnóstico para los hombres & 0.1 \\
$\mu$ & Mortalidad por causas ajenas al virus & 0.002 \\
$\omega$ & Mortalidad asociada con el virus en diagnosticados & 0.0001 \\
$\theta$ & Mortalidad asociada con el virus en no diagnosticados & 0.0005 \\
$u_{1}$ & Proporción de adopción de MP en relaciones heterosexuales & {$[0,1]$} \\
$u_{2}$ & Proporción de adopción de MP en relaciones homosexuales & {$[0,1]$} \\
\hline \hline
\end{tabular}

Con las condiciones iniciales no negativas $x_{i}(0)=x_{i}^{0}, y_{i}(0)=y_{i}^{0}$ con $i=1,2,3$ y donde $\delta_{1}=\gamma_{1}+\mu+\theta, \delta_{2}=\mu+\omega, \delta_{3}=\gamma_{2}+\mu+\theta ; \Delta_{1}>0$, $\Delta_{2}>0, \beta_{1}>0, \beta_{2}>0, \beta_{3}>0, \gamma_{1}>0, \gamma_{2}>0, \mu>0, \omega>0, \theta>0 \mathrm{y}$ $0 \leqslant u_{1}, u_{2} \leqslant 1$. La expresión del sistema es relativamente sencilla en relación con las expresiones no lineales que involucra. Es posible comprobase que satisface las condiciones de existencia y unicidad de soluciones en $\mathbb{R}^{6}[17,35]$. Más aún el sistema (1) está definido en el conjunto positivamente invariante,

$$
\Omega=\left\{\left(x_{1}, x_{2}, x_{3}, y_{1}, y_{2}, y_{3}\right) \in \mathbb{R}^{6}: 0 \leq x_{1}, x_{2}, x_{3} \leq \frac{\Delta_{1}}{\mu}, 0 \leq y_{1}, y_{2}, y_{3} \leq \frac{\Delta_{2}}{\mu}\right\} .
$$

En efecto, las soluciones $x_{1}(t)$ son no negativas puesto que $\left.\frac{d x_{1}}{d t}\right|_{x_{1}=0}=\Delta_{1}>0$ para todo $\Delta_{1}>0$, por cuanto $x_{1}(t)$ es no negativa, particularmente para $t \rightarrow+\infty$. Similarmente, teniendo en cuenta el 
dominio definido para los parámetros y las condiciones iniciales no negativas, se tiene que

$$
\left.\frac{d x_{2}}{d t}\right|_{x_{2}=0}=\beta_{1}\left(1-u_{1}\right) x_{1} y_{2} \geq\left. 0 \quad \mathrm{y} \quad \frac{d x_{3}}{d t}\right|_{x_{3}=0}=\gamma_{1} x_{2} \geq 0,
$$

por lo tanto, $x_{2}(t)$ y $x_{3}(t)$ son también no negativas. Para ver que las soluciones $x_{1}(t), \quad x_{2}(t) \quad y \quad x_{3}(t)$ están acotadas superiormente, sea $N_{x}(t)=x_{1}(t)+x_{2}(t)+x_{3}(t)$, de donde

$$
\frac{d N_{x}}{d t}=\Delta_{1}-\mu N_{x}-\omega x_{3}<\Delta_{1}-\mu N_{x}
$$

Por lo tanto, $x(t) \leq \frac{\Delta_{1}}{\mu}$, en particular para $t \rightarrow+\infty$, de donde se concluye directamente que $x_{1} \leq \frac{\Delta_{1}}{\mu}, 0 \leq x_{2} \leq \frac{\Delta_{1}}{\mu}$ y $0 \leq x_{3} \leq \frac{\Delta_{1}}{\mu}$. Un argumento similar permite probar para las variables $y_{1}(t), y_{2}(t)$ y $y_{3}(t)$. El conjunto $\Omega$ es importante porque permite garantizar que las soluciones del sistema no se hacen negativas y tampoco escapan al infinito, es decir que constituye un conjunto donde las soluciones del sistema tienen sentido biológico.

\subsection{Soluciones constantes}

Para determinar las soluciones constantes o puntos de equilibrio del sistema se resuelve el sistema algebraico:

$$
\left\{\begin{array}{l}
\Delta_{1}-\beta_{1}\left(1-u_{1}\right) x_{1} y_{2}-\mu x_{1}=0 \\
\beta_{1}\left(1-u_{1}\right) x_{1} y_{2}-\delta_{1} x_{2}=0 \\
\gamma_{1} x_{2}-\delta_{2} x_{3}=0 \\
\Delta_{2}-\beta_{2}\left(1-u_{1}\right) x_{2} y_{1}-\beta_{3}\left(1-u_{2}\right) y_{1} y_{2}-\mu y_{1}=0 \\
\beta_{2}\left(1-u_{1}\right) x_{2} y_{1}+\beta_{3}\left(1-u_{2}\right) y_{1} y_{2}-\delta_{3} y_{2}=0 \\
\gamma_{2} y_{2}-\delta_{2} y_{3}=0 .
\end{array}\right.
$$

Con lo que se puede demostrar que el punto de equilibrio trivial (ausencia de virus) corresponde a $E_{0}=\left(\frac{\Delta_{1}}{\mu}, 0,0, \frac{\Delta_{2}}{\mu}, 0,0\right)$, mientras que el único equilibrio no trivial con sentido epidemiológico, es decir, contenido en $\Omega$, 
$E_{1}=\left(\bar{x}_{1}, \bar{x}_{2}, \bar{x}_{3}, \bar{y}_{1}, \bar{y}_{2}, \bar{y}_{3}\right)$, dado por las coordenadas:

$$
\begin{aligned}
\bar{x}_{1} & =\frac{\Delta_{1}}{\beta_{1}\left(1-u_{1}\right) \bar{y}_{2}+\mu}>0 \\
\bar{x}_{2} & =\frac{\beta_{1}\left(1-u_{1}\right) \Delta_{1}}{\delta_{1}\left(\beta_{1}\left(1-u_{1}\right) \bar{y}_{2}+\mu\right)} \bar{y}_{2}>0 \\
\bar{x}_{3} & =\frac{\gamma_{1} \beta_{1}\left(1-u_{1}\right) \Delta_{1}}{\delta_{1} \delta_{2}\left(\beta_{1}\left(1-u_{1}\right) \bar{y}_{2}+\mu\right)} \bar{y}_{2}>0 \\
\bar{y}_{1} & =\frac{\Delta_{2} \delta_{1}\left(\beta_{1}\left(1-u_{1}\right) \bar{y}_{2}+\mu\right)}{\Delta_{1} \beta_{1} \beta_{2}\left(1-u_{1}\right)^{2} \bar{y}_{2} \beta_{3}\left(1-u_{2}\right) \bar{y}_{2}+\mu}>0 \\
\bar{y}_{2} & =\frac{\sqrt{p_{1}^{2}+4 p_{2} p_{0}}}{2 p_{2}}-\frac{p_{1}}{2 p_{2}} \\
\bar{y}_{3} & =\frac{\delta_{2}}{\gamma_{2}} \bar{y}_{2},
\end{aligned}
$$

donde $p_{0}, \quad p_{1}$ y $p_{2}$ son los coeficientes de la ecuación cuadrática $-p_{2} y_{2}^{2}-p_{1} y_{2}+p_{0}=0$ dados por

$$
\begin{aligned}
& p_{2}=\beta_{1}\left(1-u_{1}\right) \beta_{3}\left(1-u_{2}\right) \\
& p_{1}=\left(R_{g}-1\right) \beta_{1}\left(1-u_{1}\right)\left[\frac{\left(R_{m}+1\right) \beta_{3}\left(1-u_{2}\right)}{\left(R_{g}-1\right) \beta_{1}\left(1-u_{1}\right)}-1\right] \\
& p_{0}=\left(R_{0}-1\right),
\end{aligned}
$$

y con

$$
\begin{array}{ll}
R_{h}\left(u_{1}\right)=\frac{\beta_{2}\left(1-u_{1}\right) \Delta_{2}}{\delta_{3} \mu} & R_{m}\left(u_{1}\right)=\frac{\beta_{1}\left(1-u_{1}\right) \Delta_{1}}{\delta_{1} \mu} \\
R_{g}\left(u_{2}\right)=\frac{\beta_{3}\left(1-u_{2}\right) \Delta_{2}}{\delta_{3} \mu} & R_{0}\left(u_{1}, u_{2}\right)=R_{h}\left(u_{1}\right) R_{m}\left(u_{1}\right)+R_{g}\left(u_{2}\right) .
\end{array}
$$

En el anexo se muestra una deducción detallada de los equilibrios, proceso que lleva a concluir que la solución no trivial $E_{1}$ tiene sentido biológico siempre que $R_{0}>1$ y se tengan las condiciones:

$$
R_{g}>1 \quad \text { y } \quad \frac{\left(R_{m}+1\right) \beta_{3}\left(1-u_{2}\right)}{\left(R_{g}-1\right) \beta_{1}\left(1-u_{1}\right)}>1 .
$$

\subsection{Estabilidad local en ausencia de infección}

Mediante el proceso de linealización clásico de la Teoría de Sistemas Dinámicos [35], se llega al sistema lineal:

$$
\frac{d \mathbf{p}}{d t}=\mathbf{J p}
$$


con $\mathbf{p}=\left(x_{1}, x_{2}, x_{3}, y_{1}, y_{2}, y_{3}\right)$ y $\mathbf{J}$ es la matriz jacobiana del sistema (1) dada por:

$$
\mathbf{J}=\left(\begin{array}{cccccc}
m_{11} & 0 & 0 & 0 & m_{15} & 0 \\
m_{21} & m_{22} & 0 & 0 & m_{25} & 0 \\
0 & m_{32} & m_{33} & 0 & 0 & 0 \\
0 & m_{42} & 0 & m_{44} & m_{45} & 0 \\
0 & m_{52} & 0 & m_{54} & m_{55} & 0 \\
0 & 0 & 0 & 0 & m_{65} & m_{66}
\end{array}\right)
$$

en la cual,

$$
\begin{array}{ll}
m_{11}=-\beta_{1}\left(1-u_{1}\right) y_{2}-\mu-\lambda & m_{15}=-\beta_{1}\left(1-u_{1}\right) x_{1} \\
m_{21}=\beta_{1}\left(1-u_{1}\right) y_{2}-\lambda & m_{22}=-\delta_{1}-\lambda \\
m_{25}=\beta_{1}\left(1-u_{1}\right) x_{1} & m_{32}=\gamma_{1} \\
m_{33}=-\delta_{2}-\lambda & m_{42}=-\beta_{2}\left(1-u_{1}\right) y_{1} \\
m_{44}=-\beta_{2}\left(1-u_{1}\right) x_{2}-\beta_{3}\left(1-u_{2}\right) y_{2}-\mu-\lambda & m_{45}=-\beta_{3}\left(1-u_{2}\right) y_{1} \\
m_{52}=\beta_{2}\left(1-u_{1}\right) y_{1} & m_{65}=\gamma_{1} \\
m_{54}=\beta_{2}\left(1-u_{1}\right) x_{2}+\beta_{3}\left(1-u_{2}\right) y_{2} & m_{66}=-\delta_{2}-\lambda . \\
m_{55}=\beta_{3}\left(1-u_{2}\right) y_{1}-\delta_{3}-\lambda &
\end{array}
$$

La ecuación característica es dada por:

$$
\left(\delta_{2}+\lambda\right)^{2}\left[\lambda^{4}+a_{3} \lambda^{3}+a_{2} \lambda^{2}+a_{1} \lambda+a_{0}\right]=0
$$

donde

$$
\begin{aligned}
a_{0}= & \delta_{1}\left(\beta_{1}\left(1-u_{1}\right) y_{2}+\mu\right)\left[\beta_{3}\left(1-u_{2}\right) y_{1}\left(\beta_{2}\left(1-u_{1}\right) x_{2}+\beta_{3}\left(1-u_{2}\right) y_{2}\right)\right. \\
& \left.+\left(\delta_{3}-\beta_{3}\left(1-u_{2}\right) y_{1}\right)\left(\beta_{2}\left(1-u_{1}\right) x_{2}+\beta_{3}\left(1-u_{2}\right) y_{2}+\mu\right)\right] \\
& -\beta_{1} \beta_{2}\left(1-u_{1}\right)^{2} x_{1} \mu^{2} \\
a_{1}= & \left(\beta_{1}\left(1-u_{1}\right) y_{2}+\mu+\delta_{1}\right)\left(\beta_{2}\left(1-u_{1}\right) x_{2}+\beta_{3}\left(1-u_{2}\right) y_{2}\right) \beta_{3}\left(1-u_{2}\right) y_{1} \\
& +\left(\delta_{3}-\beta_{3}\left(1-u_{2}\right) \delta_{1}\right)\left(\beta_{2}\left(1-u_{1}\right) x_{2}+\beta_{3}\left(1-u_{2}\right) y_{2}+\mu\right) \\
& -2 \beta_{1} \beta_{2}\left(1-u_{1}\right)^{2} y_{1} \mu+\left(\beta_{1}\left(1-u_{1}\right) y_{1}+\mu\right)\left(\beta_{2}\left(1-u_{1}\right) x_{2}\right. \\
& \left.+\beta_{3}\left(1-u_{2}\right) y_{2}+\mu-\beta_{3}\left(1-u_{2}\right) y_{1}+\delta_{3}\right) \delta_{1}
\end{aligned}
$$




$$
\begin{aligned}
a_{2}= & \left(\delta_{3}-\beta_{3}\left(1-u_{2}\right) y_{1}\right)\left(\beta_{2}\left(1-u_{1}\right) x_{2}+\beta_{3}\left(1-u_{2}\right) y_{2}+\mu\right) \\
& -\beta_{1} \beta_{2}\left(1-u_{1}\right)^{2} x_{1} y_{1}+\left(\beta_{1}\left(1-u_{1}\right) y_{2}+\mu+\delta_{1}\right)\left(\beta_{2}\left(1-u_{1}\right) x_{2}\right. \\
& \left.+\beta_{3}\left(1-u_{2}\right) y_{2}+\mu-\beta_{3}\left(1-u_{2}\right) y_{1}+\delta_{3}\right) \\
& +\left(\beta_{1}\left(1-u_{1}\right) y_{2}+\mu\right) \delta_{1} \\
a_{3}= & \beta_{2}\left(1-u_{1}\right) x_{2}+\beta_{3}\left(1-u_{2}\right) y_{2}+2 \mu-\beta_{3}\left(1-u_{2}\right) y_{1}+\delta_{3} \\
& +\beta_{1}\left(1-u_{1}\right) y_{1}+\delta_{1} .
\end{aligned}
$$

Para que el polinomio característico tenga raíces con parte real negativa se requiere que $a_{1}>0, a_{3}>0, a_{4}>0$ y $a_{1} a_{2} a_{3}>a_{3}^{2}+a_{1}^{2} a_{4}$ como establece el criterio de Routh-Hurwitz [18].

En particular, para el punto de equilibrio trivial $E_{0}$ se obtiene el siguiente resultado:

Proposición 2.1 El punto de equilibrio trivial $E_{0}=\left(\frac{\Delta_{1}}{\mu}, 0,0, \frac{\Delta_{2}}{\mu}, 0,0\right)$ del sistema (1) es local y asintóticamente estable si y sólo si $R_{0}<1$.

Demostración. En el equilibrio trivial $E_{0}$ se tiene la ecuación característica:

$$
p(\lambda)=\frac{1}{\mu^{2}}(\lambda+\mu)^{2}\left(\lambda+\delta_{2}\right)^{2}\left(a_{2} \lambda^{2}+a_{1} \lambda+a_{0}\right),
$$

donde

$$
a_{2}=\mu^{2}, a_{1}=\mu^{2} \delta_{3}\left(1-R_{g}\right)+\mu^{2} \delta_{1} \text { y } a_{0}=\mu^{2} \delta_{1} \delta_{3}\left(1-R_{0}\right) .
$$

Evidentemente hay dos valores propios negativos de multiplicidad algebraica dos, los otros dos valores propios están dados por la expresión cuadrática, usando el Criterio de Routh-Hurwitz para $k=2$, se puede garantizar que los otros dos valores propios tendrán parte real negativa cuando $a_{0}>0$ y $a_{1}>0$. Note que como $R_{g}\left(u_{2}\right)=\frac{\beta_{3}\left(1-u_{2}\right) \Delta_{2}}{\delta_{3} \mu} \mathrm{y}$ $R_{0}\left(u_{1}, u_{2}\right)=R_{h}\left(u_{1}\right) R_{m}\left(u_{1}\right)+R_{g}\left(u_{2}\right)$, entonces $R_{0}<1$ garantiza automáticamente que $R_{g}<1$. Por lo tanto $a_{0}>0$ y $a_{1}>0$ si y sólo si $R_{0}<1$, con lo cual queda probada la proposición.

El análisis de estabilidad del punto de equilibrio no trivial no es fácil de efectuar, debido a la complejidad que adoptan los coeficientes del polinomio característico, sin embargo, con base en el comportamiento usual en la teoría que rodea el número básico de reproducción en epidemiología [16], se puede conjeturar que cuando $R_{0}>1$, se tiene que el equilibrio no 
trivial $E_{1}$ es local y asintóticamente estable; es decir, que se satisfarán las condiciones: $a_{1}>0, a_{3}>0, a_{4}>0$ y $a_{1} a_{2} a_{3}>a_{3}^{2}+a_{3}^{2} a_{4}$. Sin embargo, otros fenómenos como la posibilidad de bifurcación hacia atrás pueden ser estudiados en un análisis más profundo del modelo y que va más allá de los objetivos de este trabajo. En las simulaciones se ilustrará que efectivamente los escenarios con $R_{0}>1$ conllevan a un equilibrio no trivial que es asintóticamente estable, pero en ningún caso las simulaciones configuran una demostración rigurosa de lo conjeturado.

\subsection{Umbrales de control con medidas preventivas}

El Número Básico de Reproducción $R_{0}$ se define como el número de casos secundarios que produce un infectado en una población enteramente susceptible [16], para el sistema (1) se dedujo al estudiar la solución constante en presencia de virus y está dado por $R_{0}\left(u_{1}, u_{2}\right)=R_{h}\left(u_{1}\right) R_{m}\left(u_{1}\right)+R_{g}\left(u_{2}\right)$, que de forma explícita es

$$
R_{0}\left(u_{1}, u_{2}\right)=\frac{\beta_{2}\left(1-u_{1}\right) \Delta_{2}}{\delta_{3} \mu} \frac{\beta_{1}\left(1-u_{1}\right) \Delta_{1}}{\delta_{1} \mu}+\frac{\beta_{3}\left(1-u_{2}\right) \Delta_{2}}{\delta_{3} \mu} .
$$

Puede notarse la influencia (efectos aditivos y multiplicativos) de cada una de las vías de transmisión consideradas en el modelo, en efecto, $R_{h}$ y $R_{m}$ corresponden a los aportes hechos por hombres y mujeres mediante los contactos heterosexuales, mientras que el sumando $R_{g}$ corresponde al aporte hecho por los hombres mediante los contactos homosexuales. En la Figura 2 se muestra la gráfica de la función $R_{0}\left(u_{1}, u_{2}\right)$ para los valores de los parámetros dados en la Tabla 1. La figura permite apreciar que los menores valores del $R_{0}$ se obtiene para valores de $u_{1}$ y $u_{2}$ muy cercanos a 1 , es decir, cuando la adopción de las medidas preventivas se hace por la totalidad de la población. Una forma de evidenciar esto analíticamente, por lo menos de forma aproximada, es estudiando la adopción de medidas preventivas que se requiere, considerando prevención solo en relaciones heterosexuales o solo en relaciones homosexuales exclusivamente. 


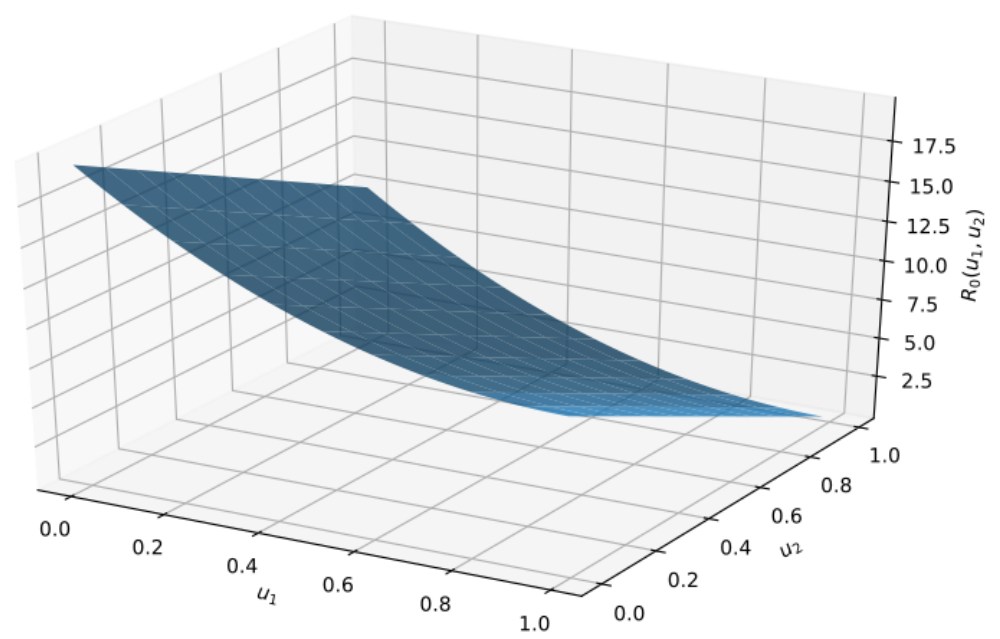

Figura 2: Número básico de reproducción $R_{0}\left(u_{1}, u_{2}\right)$, con los valores de los parámetros que se muestran en la Tabla 1 .

Se ha probado que el equilibrio libre de infección $E_{0}$ es local y asintóticamente estable si y sólo si $R_{0}\left(u_{1}, u_{2}\right)<1$. El objetivo en este punto es determinar umbrales $U_{H}$ y $U_{G}$ de control mediante medidas preventivas exclusivamente para las relaciones heterosexuales y homosexuales respectivamente.

Umbral de prevención para relaciones heterosexuales. Para determinar $U_{H}$, considere ausencia del control en las relaciones homosexuales, $u_{2}=0 ;$ así, $R_{0}\left(u_{1}, 0\right)<1$, es decir,

$$
R_{0}\left(u_{1}, 0\right)=R_{h}\left(u_{1}\right) R_{m}\left(u_{1}\right)+R_{g}(0)<1
$$

Despejando $u_{1}$ se tiene:

$$
u_{1}>U_{H}=\frac{\mu}{\Delta_{1}} \sqrt{\frac{\delta_{1} \delta_{3}}{\beta_{1} \beta_{3}}\left(1-R_{g}(0)\right)}+1>1,
$$

expresión que solo tiene sentido si $R_{g}(0)<1$ y en ese caso, esta cantidad siempre es mayor que 1 , por lo tanto no es posible llevar el sistema al equilibrio libre de infección exclusivamente con medidas preventivas en las relaciones heterosexuales. 
Umbral de prevención para relaciones homosexuales. Del mismo modo, en ausencia de control en la población heterosexual, $u_{1}=0$, la condición $R_{0}\left(0, u_{2}\right)<1$ implica

$$
R_{0}\left(0, u_{2}\right)=R_{h}(0) R_{m}(0)+R_{g}\left(u_{2}\right)<1,
$$

y al despejar $u_{2}$ se obtiene:

$$
u_{2}>U_{G}=\frac{R_{0}(0,0)-1}{\mathcal{R}_{g}(0)}>1 .
$$

Esta cantidad es siempre mayor que 1, provisto $R_{0}(0,0)>1$, y en consecuencia tampoco es posible llevar el sistema al equilibrio libre de infección $E_{0}$ sólo con medidas preventivas en las relaciones homosexuales.

Con lo que se tiene hasta ahora, se puede afirmar que para eliminar la transmisión de la infección, acudiendo solo a medidas preventivas, se requiere que casi la totalidad de la población, sin importar la orientación sexual, debe adoptar las medidas preventivas como una práctica usual. La simulación de diversos escenarios en la próxima sección, mostrará evidencias de esta afirmación.

\subsection{Simulación}

El modelo para la transmisión del VIH con medidas preventivas fue simulado con las condiciones iniciales hipotéticas $x_{1}(0)=250, x_{2}(0)=0$, $x_{3}(0)=0, y_{1}(0)=249, y_{2}(0)=1$ y $y_{3}(0)=0$, y valores para los parámetros descritos en la Tabla 1. Las simulaciones fueron hechas en un programa de computación científica con un esquema Runge-Kutta de orden cuatro, el cual es ampliamente conocido que permite converger a las soluciones del sistema con muy buenos márgenes de error. En cada escenario simulado se muestran seis (6) curvas, que corresponden a diferentes proporciones de adopción de las medidas preventivas $u_{1}$ y $u_{2}$.

El escenario que se ilustra en la Figura 3 muestra el comportamiento cuando se hace uso de medidas preventivas solo en las relaciones homosexuales, es decir $u_{1}=0$, con lo que se obtuvieron los valores de $R_{0}$ que se describen en la leyenda. Para una mejor interpretación de la gráfica, se debe tener en cuenta los valores menores de $u_{2}$, dan origen a las curvas con menores niveles de susceptibles y mayores niveles de infectados. Se puede evidenciar entonces, como ya se mostró en la sección anterior, que no es posible controlar la transmisión solo con el uso de medidas preventivas en las relaciones homosexuales, ni siquiera con una adopción del $100 \%$.

Rev.Mate.Teor.Aplic. (ISSN print: 1409-2433; online: 2215-3373) Vol. 25(2): 293-318, Jul-Dec 2018 

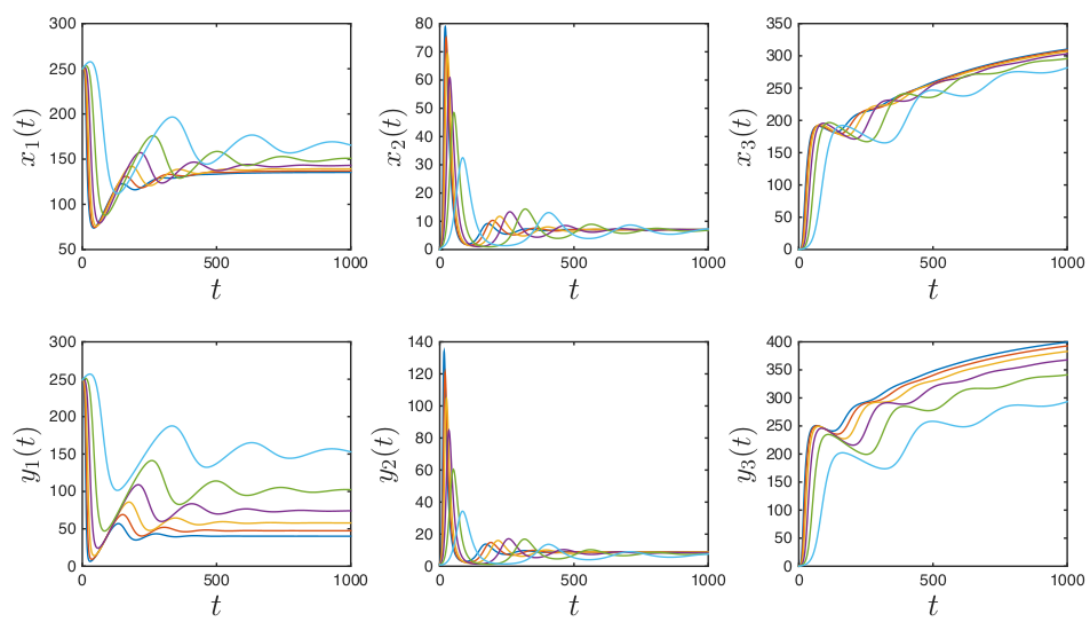

Figura 3: Simulación considerando solo prevención en las relaciones homosexuales; es decir, $u_{1}=0$ y $u_{2}=\left(\begin{array}{lllll}0 & 0.2, & 0.4,0.6,0.8,1\end{array}\right)$ y los parámetros de la Tabla 1 , con lo que se obtiene $R_{0}=$ $(19.7501,17.7989,15.8477,13.8965,11.9453,9.9941)$, respectivamente.
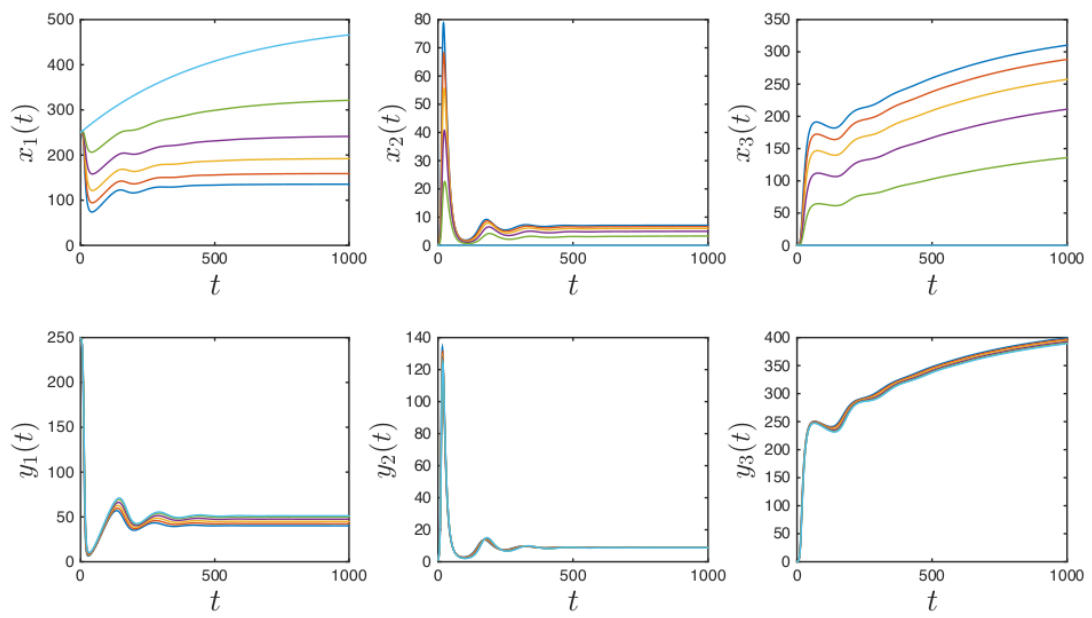

Figura 4: Simulación considerando solo prevención en las relaciones heterosexuales es decir, $u_{2}=(0,0.2,0.4,0.6,0.8,1)$. En este caso los valores del $R_{0}$ son $R_{0}=(19.7501,16.1523,13.3540,11.3551,10.1559,9.7561)$ respectivamente. 
En el escenario de la Figura 4, se muestra el uso de medidas preventivas por solo en las relaciones heterosexuales, lo que implica $u_{2}=0$. Si bien la infección se encuentra presente en ambas poblaciones, es evidente que tiene un mayor impacto sobre los hombres que sobre las mujeres, hecho que se debe, evidentemente, a la ausencia de medidas preventivas en las relaciones sexuales que involucran solo hombres. Nuevamente se puede observar que ni siquiera una adopción del $100 \%$ de las medidas preventivas en las relaciones heterosexuales, puede controlar plenamente la transmisión de la infección.
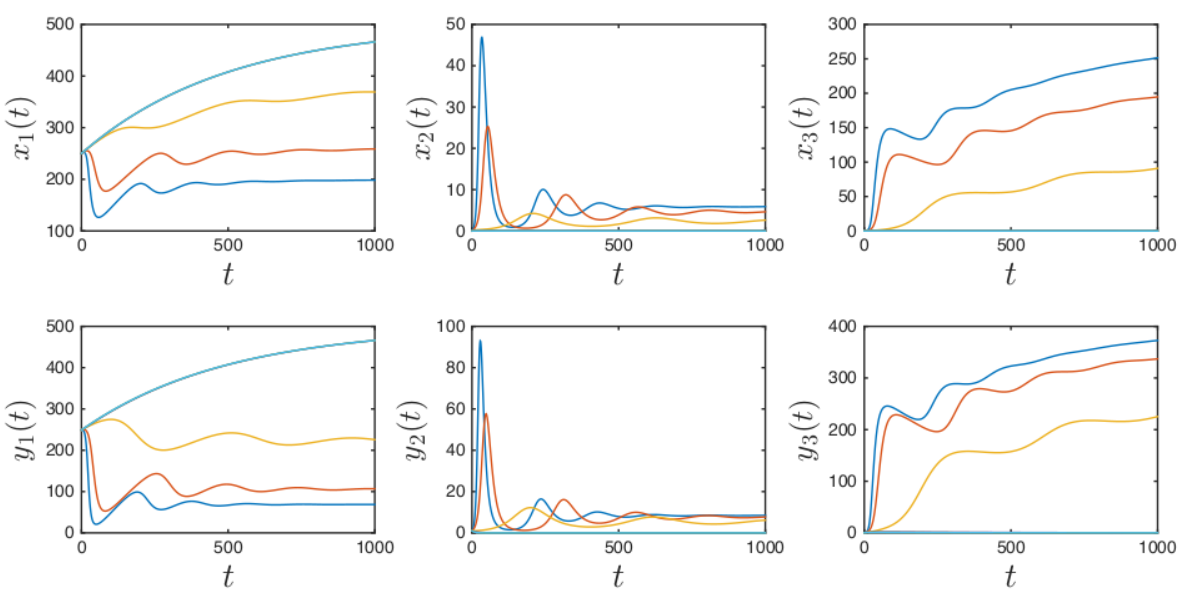

Figura 5: Simulación considerando igual nivel de adopción de las medidas preventivas en las relaciones sexuales; es decir, $u_{1}=u_{2}=$ $(0.4,0.6,0.8,0.9,0.95,1)$, con lo que se obtuvo $R_{0}=$ $(9.4515,5.5015,2.3510,1.0756,0.5128,0)$ respectivamente.

En la Figura 5 se considera que $u_{1}=u_{2}$; es decir, que los niveles de adopción de las medidas preventivas son iguales en las relaciones heterosexuales y las homosexuales. Una situación interesante que se presenta en este escenario es que para $u_{1}=u_{2}=0.95$ y para $u_{1}=u_{2}=1$ se obtienen valores de $R_{0}$ menores que 1 , en efecto $R_{0}=0.5128$ y $R_{0}=0$, respectivamente. Ambas curvas se han representado en azul claro en la Figura 5 pero se superponen una sobre la otra. Este hecho pone en evidencia que los niveles de adopción de las medidas preventivas tienen que ser muy cercanos al 100\%, para que se logre obtener un equilibrio libre de infección localmente asintóticamente estable. 


\section{Resultados y conclusiones}

Mediante el modelo propuesto se interpreta la dinámica de transmisión del VIH con un modelo original de ecuaciones diferenciales ordinarias para una población bisexual sin transmisión del virus por relaciones homosexuales entre mujeres. Se estudió la estabilidad local del equilibrio en ausencia de virus a partir de el Número Básico de Reproducción $R_{0}$, de donde se derivan los umbrales de control por medidas preventivas en las relaciones heterosexuales $U_{H}$ y en las relaciones homosexuales $U_{G}$. La forma explícita de los umbrales permite concluir además que no es posible eliminar la transmisión del virus considerando cada caso de forma exclusiva.

Se simula el modelo numéricamente con el programa para ilustrar diferentes escenarios de control mediante medidas preventivas e ilustrar la existencia de por lo menos un equilibrio en presencia de la infección que es localmente asintóticamente estable cuando el número básico de reproducción es mayor que 1.

Las medidas preventivas consideradas resultan eficaces para controlar la transmisión del virus, únicamente cuando son aplicadas por toda la población, sin tener en cuenta diferencias de orientación sexual, ya que tal diferenciación muestra no tener impacto ni benéfico ni perjudicial. El modelo muestra que los hombres se ven más afectados debido a que enfrentan dos vías de contacto sexual por las cuales puede adquirir el virus. Evidentemente la incorporación de supuestos diferentes, como la transmisión vertical o la parenteral, debe dar origen a escenarios de transmisión muy ricos y considerablemente diferentes a los ilustrados acá.

Considerar la población diferenciada y la orientación sexual, hacen que el sistema se vuelva de difícil controlabilidad, ya que en la práctica no es posible garantizar que entre el $95 \%$ y $100 \%$ de la población adopte medidas preventivas; por esto, es importante investigar otras estrategias de control que permitan reducir la transmisión del VIH en las poblaciones susceptibles.

Si en algún momento se quiere adaptar modelos como el descrito a poblaciones concretas, con el fin de obtener predicciones y tomar decisiones, se debe garantizar que se satisfagan los supuestos del modelo y conocer valores reales para los parámetros, lo que podría ayudar a una mejor interpretación del comportamiento de la enfermedad y a la búsqueda de soluciones efectivas y a largo plazo.

Rev.Mate.320.Aplic. (ISSN print: 1409-2433; online: 2215-3373) Vol. 25(2): 293-318, Jul-Dec 2018 


\section{Referencias}

[1] Akin, M.; Fernández, M.; Bowen, G.; Warren, J. (2008) "HIV risk behaviors of Latin American and Caribbean men who have sex with men in Miami, Florida, USA", Revista Panamericana de Salud Pública 23(5): $341-348$.

[2] Attia, S.; Egger, M.; Müller, M.; Zwahlen, M.; Low, N. (2009) "Sexual transmission of HIV according to viral load and antiretroviral therapy: systematic review and meta-analysis", AIDS 23(11): 1397-1404.

[3] Barros, T.; Barreto, D.; Pérez, F.; Santander, R.; Yépez, E.; AbadFranch, F.; Aguilar, M. (2001) "Un modelo de prevención primaria de las enfermedades de transmisión sexual y del VIH/SIDA en adolescentes", Revista Panamericana de Salud Pública 10(2): 86-94.

[4] Bowong, S. (2010) "Optimal control of the transmission dynamics of tuberculosis", Nonlinear Dynamics 61(4):729-748.

[5] Caetano, M.A.L.; Yoneyama, T. (2001) "Optimal and sub-optimal control in Dengue epidemics", Optimal Control Applications and Methods 22: 63-73.

[6] Casals, M.; Guzmán, K.; Caylà, J. (2009) "Modelos matemáticos utilizados en el estudio de las enfermedades transmisibles", Revista Española de Salud Pública 83(5): 689-695.

[7] Cassels, S.; Clark, S.; Morris, M. (2008) "Mathematical models for HIV transmission dynamics: tools for social and behavioral science research", Journal of Acquired Immune Deficiency Syndromes 47(1): $1-11$.

[8] Culshaw, R.; Ruan, S.; Spiteri, R. (2004) "Optimal HIV treatment by maximising immune response", Mathematical Biology 48(5): $545-562$.

[9] Derouich, M.; Boutayeb, A. (2008) "An avian influenza mathematical model", Applied Mathematical Sciences 2(36): 1749-1760.

[10] Desai, K.; Boily, M.; Garnett, G.; Mâsse, B.; Moses, S.; Bailey, R. (2006) "The role of sexually transmitted infections in male circumcision effectiveness against HIV - insights from clinical trial simulation", Emerging Themes in Epidemiology 3(19): 1-16. 
[11] Deschamps, M.; Noel, F.; Bonhomme, J.; Dévieux, J.; Saint-Jean, G.; Zhu, Y.; Wright, P.; Pape, J.; Malow, R. (2009) "Prevention of mother-to-child transmission of HIV in Haiti", Revista Panamericana de Salud Pública 25(1): 24-30.

[12] Ferreyra, G.; Pascal, J. (1999) "Control óptimo determinista vía programación dinámica”, Divulgaciones Matemáticas 7(2): 167-185.

[13] Fung, I.; Gambhir, M.; Sighem, A.; Wolf, F.; Garnett, G. (2010) "Superinfection with a heterologous HIV strain per se does not lead to faster progression", Mathematical Biosciences 224(1): 1-9.

[14] Gómez, M. (2008) "Comparación de tres estrategias de tamizaje para la prevención de la infección perinatal por VIH en Colombia: análisis de decisiones", Revista Panamericana de Salud Pública 24(4): $256-264$.

[15] Hattaf, K.; Rachik, M.; Saadi, S.; Tabit, Y.; Yousfi, N. (2009) "Optimal control of tuberculosis with exogenous reinfection", Applied Mathematical Sciences 3(5): 231-240.

[16] Heesterbeek, J.A.P. (2002) "A brief history of $R_{0}$ and a recipe for its calculation", Acta biotheoretica 50(3): 189-204.

[17] Hirsch, M.W.; Smale, S.; Devaney, R.L. (2012) Differential Equations, Dynamical Systems, and an Introduction to Chaos. Academic Press, New York.

[18] Hurwitz, A. (1913) "Über einen Satz des Herrn Kakeya", Tohoku Mathematical Journal, First Series 4: 89-93.

[19] Jafelice, R.; Bassanezi, R. (2007) "Curvas padrões de tratamento do HIV", Biomatemática 17: 55-64.

[20] Jafelice, R.; Barros, L.; Bassanezi, R.; Gomide, F. (2003) "Influência da AIDS na expectativa de vida de uma População", Biomatemática XIII: $75-84$.

[21] Johnson, L.; Dorrington, R. (2006) "Modelling the demographic impact of HIV/AIDS in South Africa and the likely impact of interventions", Demographic Research 14: 541-574.

[22] Joshi, H. (2002) "Optimal control of an HIV inmunology model", Optimal Control Applications and Methods 23(4): 199-213. 
[23] Jung, E.; Iwami, S.; Takeuchi, Y.; Jo, T. (2009) "Optimal control strategy for prevention of avian influenza pandemic", Journal of Theoretical Biology 260(2): 220-229.

[24] Kaplan, E.; Cramton, P.; Paltiel, D. (1989) "Nonrandom mixing models of HIV transmission", in: C. Castillo-Chávez (Ed.) Mathematical and Statistical Approaches to AIDS Epidemiology, Springer-Verlag, New York: 1-15.

[25] Karrakchou, J.; Rachik, M.; Gourari, S. (2006) "Optimal control and infectiology: Application to an HIV/AIDS model", Applied Mathematics and Computation 117(2): 807-818.

[26] Kimbir, A.; Oduwole, H. (2008) "A mathematical model of HIV/AIDS transmission dynamics considering counselling and antiretroviral therapy", Journal of Modern Mathematics and Statistics 2(5): 166-169.

[27] Lasry, A.; sansom, S.L.; Wolitski, R.J.; Green, T.A.; Borkowf, C.B.; Patel, P.; Mermin, J. (2014) "HIV secual transmission risk among serodiscordant couples: assesing the effects of combining prevention strategies", AIDS 28(10): 1521-1529.

[28] Mesa-Mazo, M.; Vergaño-Salazar, J.; Sánchez-Botero, C.; MuñozLoaiza, A. (2010) "Modelo matemático para la dinámica de transmisión del VIH/SIDA en una población sexualmente activa", Revista de Salud Pública 12(2): 308-316.

[29] Montesinos-López, O.; Hernández-Suárez, C. (2007) "Modelos matemáticos para enfermedades infecciosas", Salud Pública 49(3): $218-226$.

[30] Muñoz, L. (2007) Modelado Matematico del Dengue Clásico. Tesis de Doctorado, Facultad de Ciencias Físico Matemáticas, Benemérita Universidad Autónoma de Puebla, México.

[31] Nanda, S.; Moore, H.; Lenhart, S. (2007) "Optimal control of treatment in a mathematical model of chronic myelogenous leukemia", Mathematical Biosciences 210(1): 143-156.

[32] Newton, E.; White, F.; Sokal, D.; King, T.; Forsythe, S. (1994) "Modelos para el estudio de la epidemia del SIDA en el Caribe de habla inglesa", Boletín de la Oficina Sanitaria Panamericana 117(4): 296-306. 
[33] ONUSIDA (2017) "Hoja informativa-día mundial del SIDA de 2017", en: http://www. unaids.org/sites/default/files/media_ asset/UNAIDS_FactSheet_es.pdf, consultado el 07/05/2018.

[34] Pérez, J.L; Toro-Zapata, H.D. (2014) "Modeling the cytotoxic immune response effects on human immunodeficiency virus", Visión Electrónica 8(1): 54-62.

[35] Perko, L. (1996) Differential Equations and Dynamical Systems. Springer-Verlag, New York.

[36] Podzamczer, D.; Panel de expertos de GESIDA y Plan Nacional sobre el Sida (2008) "Tratamiento de las infecciones oportunistas en pacientes adultos y adolescentes infectados por el virus de la inmunodeficiencia humana en la era del tratamiento antirretroviral de gran actividad. Recomendaciones del Grupo de Estudio del Sida (GESIDA)/Plan Nacional sobre el Sida", Enfermedades Infecciosas y Microbiología Clínica 26(6): 356-379.

[37] Rodríguez, J.M. (2004) "Papel de los modelos en las evaluaciones económicas en el campo sanitario", Revista de Farmacia Hospitalaria 28(4): 231-242.

[38] Rodrigues-Júnior, A.; Castilho, E. (2009) "A AIDS nas regiões de fronteira no Brasil de 1990 a 2003", Revista Panamericana de Salud Pública 25(1): 31-8.

[39] Samayoa, B.; Anderson, M.; Grazioso, C.; Rivera, B.; Harrison, M.; O'Brien, W.; Arathoon, E. (2009) "Experience of a pediatric HIV clinic in Guatemala City", Revista Panamericana de Salud Pública 25(1): $51-55$.

[40] Toro-Zapata, H.D. (2010) Modelo de la Transmisión del Dengue Clásico Durante un Brote Epidémico. Tesis de Maestría, Departamento de Biomatemáticas, Universidad del Quindío, Quindío.

[41] Toro-Zapata, H.D.; Caicedo, A. G.; Bichara, D.; Lee, S. (2014) "Role of active and inactive cytotoxic immune response in human immunodeficiency virus dynamics", Osong Public Health and Research Perspectives 5(1): $3-8$. 
[42] Toro-Zapata, H.D.; Londoño-González, C.A.; Trulijjo-Salazar, C. A. (2014) "Modelo de simulación para la infección por VIH y su interacción con la respuesta inmune citotóxica", Revista de Salud Publica 16(1): 114-127.

[43] Toro-Zapata, H.D.; Mesa-Mazo, M.; Prieto-Medellín, D. (2014) "Modelo de simulación para la transmisión del VIH y estrategias de control basadas en diagnóstico", Revista de Salud Pública 16(1): 139-152.

[44] Toro-Zapata, H.D.; Roa-Vásquez, E.; Mesa-Mazo, M.J. (2017) "Modelo estocástico para la infección con VIH de las células T CD4+ del sistema inmune", Revista de Matemática: Teoría y Aplicaciones 24(2): 287-313.

[45] Trujillo-Salazar, C.A.; Toro-Zapata, H.D. (2014) "Análisis teórico de la transmisión y el control del VIH/SIDA en un centro de reclusión", in: D. Tarzia (Ed.) VII Italian-Latin American Conference on Industrial and Applied Mathematics, Universidad Austral, Facultad de Ciencias Empresariales, Argentina: 17-26.

[46] Trujillo-Salazar. C.A.; Toro-Zapata, H.D. (2015) "Simulation model for AIDS dynamics and optimal control through antiviral treatment", in: G. Tost \& O. Vasilieva (Eds.) Analysis, Modelling, Optimization and Numerical Techniques, ICAMI, San Andres Island, Colombia: $257-270$.

[47] UNAIDS. (2017) UNAIDS Data 2017. Joint United Nations Programme on HIV/AIDS, Geneva.

[48] Valencia, C.J.; Gómez, B.N. (2003) "Evaluación de un programa de prevención primaria en VIH/SIDA para jóvenes ente 14 y 15 años", Centro de Investigaciones y Desarrollo Científico, Universidad Distrital Francisco José de Caldas Primera Edición: 61-63.

[49] Wang, Y.; Zhou, Y.; Wu, J.; Heffernan, J. (2009) "Oscillatory viral dynamics in a delayed HIV pathogenesis model", Mathematical Biosciences 219(2): 104-112.

[50] Zavaleta, C.; Mujica, J.; Ypanaqué, P.; Cueva, N. (2007) "Infecciones de transmisión sexual y VIH/SIDA en comunidades nativas de la amazonía peruana: consideraciones culturales", Revista Peruana de Medicina Experimental y Salud Pública 24(3): 315-316. 


\section{Anexo: deducción de las soluciones constantes}

De la primera ecuación del sistema algebraico (2)

$$
\Delta_{1}-\beta_{1}\left(1-u_{1}\right) x_{1} y_{2}-\mu x_{1}=0,
$$

se despeja $x_{1}$ :

$$
x_{1}=\frac{\Delta_{1}}{\beta_{1}\left(1-u_{1}\right) y_{2}+\mu} .
$$

Se reemplaza en la segunda ecuación del sistema algebraico (2),

$$
\beta_{1}\left(1-u_{1}\right) x_{1} y_{2}-\delta_{1} x_{2}=0 .
$$

El valor obtenido en (4) y despejando $x_{2}$, se tiene:

$$
x_{2}=\frac{\beta_{1}\left(1-u_{1}\right) \Delta_{1}}{\delta_{1}\left(\beta_{1}\left(1-u_{1}\right) y_{2}+\mu\right)} y_{2} .
$$

Reemplazando (5) en la tercera ecuación del sistema algebraico (2),

$$
\gamma_{1} x_{2}-\delta_{2} x_{3}=0
$$

de donde $x_{3}$ es igual a

$$
x_{3}=\frac{\gamma_{1} \beta_{1}\left(1-u_{1}\right) \Delta_{1}}{\delta_{2} \delta_{1}\left(\beta_{1}\left(1-u_{1}\right) y_{2}+\mu\right)} y_{2} .
$$

Reemplazando en la cuarta ecuación del sistema algebraico (2) a (5),

$$
\Delta_{2}-\beta_{2}\left(1-u_{1}\right) x_{2} y_{1}-\beta_{3}\left(1-u_{2}\right) y_{1} y_{2}-\mu y_{1}=0
$$

se tiene $y_{1}$

$$
y_{1}=\frac{\Delta_{2}}{\frac{\beta_{1} \beta_{2}\left(1-u_{1}\right)^{2} \Delta_{1} y_{2}}{\delta_{1}\left(\beta_{1}\left(1-u_{1}\right) y_{2}+\mu\right)}+\beta_{3}\left(1-u_{2}\right) y_{2}+\mu} .
$$

Despejando $y_{3}$ de la última ecuación del sistema algebraico (2)

$$
y_{3}=\frac{\delta_{2}}{\gamma_{2}} y_{2}
$$

y reemplazando en la quinta ecuación del sistema algebraico (2) a (5) y a (7), se tiene: 


$$
\begin{gathered}
\beta_{2}\left(1-u_{1}\right)\left(\frac{\beta_{1}\left(1-u_{1}\right) \Delta_{1}}{\delta_{1}\left(\beta_{1}\left(1-u_{1}\right) y_{2}+\mu\right)} y_{2}\right)\left(\frac{\Delta_{2}}{\frac{\beta_{1} \beta_{2}\left(1-u_{1}\right)^{2} \Delta_{1} y_{2}}{\delta_{1}\left(\beta_{1}\left(1-u_{1}\right) y_{2}+\mu\right)}+\beta_{3}\left(1-u_{2}\right) y_{2}+\mu}\right) \\
+\beta_{3}\left(1-u_{2}\right)\left(\frac{\Delta_{2}}{\frac{\beta_{1} \beta_{2}\left(1-u_{1}\right)^{2} \Delta_{1} y_{2}}{\delta_{1}\left(\beta_{1}\left(1-u_{1}\right) y_{2}+\mu\right)}+\beta_{3}\left(1-u_{2}\right) y_{2}+\mu}\right) y_{2}-\delta_{3} y_{2}=0 .
\end{gathered}
$$

Factorizando $y_{2}$,

$$
\begin{gathered}
{\left[\frac{\beta_{1} \beta_{2}\left(1-u_{1}\right)^{2} \Delta_{1} \Delta_{2}}{\beta_{1} \beta_{2}\left(1-u_{1}\right)^{2} \Delta_{1} y_{2}+\delta_{1}\left(\beta_{1}\left(1-u_{1}\right) y_{2}+\mu\right)\left(\beta_{3}\left(1-u_{2}\right) y_{2}+\mu\right)}+\right.} \\
\left.\frac{\beta_{3}\left(1-u_{2}\right) \Delta_{2} \delta_{1}\left(\beta_{1}\left(1-u_{1}\right) y_{2}+\mu\right)}{\beta_{2} \beta_{1}\left(1-u_{1}\right)^{2} \Delta_{1} y_{2}+\delta_{1}\left(\beta_{1}\left(1-u_{1}\right) y_{2}+\mu\right)\left(\beta_{3}\left(1-u_{2}\right) y_{2}+\mu\right)}-\delta_{3}\right] y_{2}=0,
\end{gathered}
$$

por lo tanto

$$
y_{2}=0
$$

Ó

$$
\frac{\beta_{2} \beta_{1}\left(1-u_{1}\right)^{2} \Delta_{1} \Delta_{2}+\beta_{3}\left(1-u_{2}\right) \Delta_{2} \delta_{1}\left(\beta_{1}\left(1-u_{1}\right) y_{2}+\mu\right)}{\beta_{1} \beta_{2}\left(1-u_{1}\right)^{2} \Delta_{1} y_{2}+\delta_{1}\left(\beta_{1}\left(1-u_{1}\right) y_{2}+\mu\right)\left(\beta_{3}\left(1-u_{2}\right) y_{2}+\mu\right)}-\delta_{3}=0 .
$$

Si $y_{2}=0$ se tiene lo siguiente:

$$
x_{1}=\frac{\Delta_{1}}{\mu}, x_{2}=0, x_{3}=0, y_{1}=\frac{\Delta_{2}}{\mu}, y_{2}=0, y_{3}=0 .
$$

Así, el punto trivial del sistema $E_{0}=\left(\frac{\Delta_{1}}{\mu}, 0,0, \frac{\Delta_{2}}{\mu}, 0,0\right)$ y corresponde al estado libre de infección. En el otro caso, simplificando (10) se tiene

$$
\begin{aligned}
& -\delta_{1} \delta_{3} \beta_{1}\left(1-u_{1}\right) \beta_{3}\left(1-u_{2}\right) y_{2}^{2}-\left(\left[\frac{\beta_{1}\left(1-u_{1}\right) \Delta_{1}}{\delta_{1} \mu}+1\right] \beta_{3}\left(1-u_{2}\right)\right. \\
& \left.-\left[\frac{\beta_{3}\left(1-u_{2}\right) \Delta_{2}}{\delta_{3} \mu}-1\right] \beta_{1}\left(1-u_{1}\right)\right) \delta_{1} \delta_{3} \mu y_{2} \\
& +\delta_{1} \delta_{3} \mu\left(\frac{\beta_{1} \beta_{2}\left(1-u_{1}\right)^{2} \Delta_{1} \Delta_{2}}{\delta_{3} \delta_{1} \mu^{2}}+\frac{\beta_{3}\left(1-u_{2}\right) \Delta_{2}}{\delta_{3} \mu}-1\right)=0
\end{aligned}
$$


Sean

$$
\begin{array}{ll}
R_{h}\left(u_{1}\right)=\frac{\beta_{2}\left(1-u_{1}\right) \Delta_{1}}{\delta_{3} \mu} & R_{m}\left(u_{1}\right)=\frac{\beta_{1}\left(1-u_{1}\right) \Delta_{1}}{\delta_{1} \mu} \\
R_{g}\left(u_{2}\right)=\frac{\beta_{3}\left(1-u_{2}\right) \Delta_{2}}{\delta_{3} \mu} & R_{0}\left(u_{1}\right)=R_{h}\left(u_{1}\right) R_{m}\left(u_{1}\right)+R_{g}\left(u_{2}\right) .
\end{array}
$$

entonces la anterior ecuación queda:

$$
\begin{gathered}
-\delta_{1} \delta_{3} \beta_{1}\left(1-u_{1}\right) \beta_{3}\left(1-u_{2}\right) y_{2}^{2}-\left(R_{g}-1\right)\left(\frac{\left(R_{m}+1\right) \beta_{3}\left(1-u_{2}\right)}{\left(R_{g}-1\right) \beta_{1}\left(1-u_{1}\right)}-1\right) \\
\beta_{1}\left(1-u_{1}\right) y_{2}+\left(R_{0}-1\right)=0,
\end{gathered}
$$

que corresponde a una ecuación cuadrática de la forma:

$$
-p_{2} y_{2}^{2}-p_{1} y_{2}+p_{0}=0
$$

donde

$$
\begin{aligned}
& p_{2}=\beta_{1}\left(1-u_{1}\right) \beta_{3}\left(1-u_{2}\right) \\
& p_{1}=\left(R_{g}-1\right) \beta_{1}\left(1-u_{1}\right)\left[\frac{\left(R_{m}+1\right) \beta_{3}\left(1-u_{2}\right)}{\left(R_{g}-1\right) \beta_{1}\left(1-u_{1}\right)}-1\right] \\
& p_{0}=\left(R_{0}-1\right) .
\end{aligned}
$$

Puede verse que si'la ecuación 3:

$$
R_{g}>1 \quad \text { y } \frac{\left(R_{m}+1\right) \beta_{3}\left(1-u_{2}\right)}{\left(R_{g}-1\right) \beta_{1}\left(1-u_{1}\right)}>1,
$$

se garantiza que el coeficiente del término lineal es negativo. Del mismo modo, si $R_{0}>1$ el coeficiente $p_{0}$ es positivo la ecuación (11) tiene soluciones de la forma:

$$
y_{2}^{ \pm}=-\frac{p_{1}}{2 p_{2}} \pm \frac{\sqrt{p_{1}^{2}+4 p_{2} p_{0}}}{-2 p_{2}} \in \mathbb{R}
$$

de donde

$$
y_{2}^{+}=-\frac{p_{1}}{2 p_{2}}+\frac{\sqrt{p_{1}^{2}+4 p_{2} p_{0}}}{2 p_{2}}, \quad y_{2}^{-}=-\frac{p_{1}}{2 p_{2}}-\frac{\sqrt{p_{1}^{2}+4 p_{2} p_{0}}}{2 p_{2}} .
$$

Luego, $y_{2}^{-}<0$ y cuando $\beta_{1}\left(1-u_{1}\right) \beta_{3}\left(1-u_{2}\right)\left(R_{0}-1\right)>0$ entonces $y_{2}^{+}>0$; por lo tanto $y_{2}^{+} \in \Omega$ si y sólo si $R_{0}>1$. 
Ahora, con $R_{0}>1$ y las condiciones dadas en (3) se obtiene finalmente el punto de equilibrio no trivial $E_{1}=\left(\bar{x}_{1}, \bar{x}_{2}, \bar{x}_{3}, \bar{y}_{1}, \bar{y}_{2}, \bar{y}_{3}\right)$, donde

$$
\begin{aligned}
\bar{x}_{1} & =\frac{\Delta_{1}}{\beta_{1}\left(1-u_{1}\right) y_{2}+\mu}>0 \\
\bar{x}_{2} & =\frac{\beta_{1}\left(1-u_{1}\right) \Delta_{1}}{\delta_{1}\left(\beta_{1}\left(1-u_{1}\right) y_{2}+\mu\right)} y_{2}>0 \\
\bar{x}_{3} & =\frac{\gamma_{1} \beta_{1}\left(1-u_{1}\right) \Delta_{1}}{\delta_{1} \delta_{2}\left(\beta_{1}\left(1-u_{1}\right) y_{2}+\mu\right)} y_{2}>0 \\
\bar{y}_{1} & =\frac{\Delta_{2} \delta_{1}\left(\beta_{1}\left(1-u_{1}\right) y_{2}+\mu\right)}{\Delta_{1} \beta_{1} \beta_{2}\left(1-u_{1}\right)^{2} y_{2} \beta_{3}\left(1-u_{2}\right) y_{2}+\mu}>0 \\
\bar{y}_{2} & =\frac{\sqrt{p_{1}^{2}+4 p_{2} p_{0}}}{2 p_{2}}-\frac{p_{1}}{2 p_{2}} \\
\bar{y}_{3} & =\frac{\delta_{2}}{\gamma_{2}} y_{2} .
\end{aligned}
$$

